\title{
Integrative microRNA-gene expression network analysis in genetic hypercalciuric stone-forming rat kidney
}

Yuchao Lu, Baolong Qin, Henglong Hu, Jiaqiao Zhang, Yufeng Wang, Qing Wang, Shaogang Wang

Background. MicroRNAs (miRNAs) influence a variety of biological functions by regulating gene expression post-transcriptionally. Aberrant miRNA expression has been associated with many human diseases. Urolithiasis is a common disease, and idiopathic hypercalciuria $(\mathrm{IH})$ is an important risk factor for calcium urolithiasis. However, miRNA expression patterns and their biological functions in urolithiasis remain unknown. Methods and Results. A multi-step approach combining microarray miRNA and mRNA expression profile and bioinformatics analysis was adopted to analyze dysregulated miRNAs and genes in genetic hypercalciuric stone-forming (GHS) rat kidneys, using normal SpragueDawley (SD) rats as controls. We identified 2418 mRNAs and 19 miRNAs as significantly differentially expressed, over 700 Gene Ontology (GO) terms and 83 KEGG pathways that were significantly enriched in GHS rats. In addition, we constructed an miRNA-gene network that suggested that rno-miR-674-5p, rno-miR-672-5p, rno-miR-138-5p and rnomiR-21-3p may play important roles in the regulatory network. Furthermore, signal-net analysis suggested that NF-kappa B likely plays a crucial role in hypercalciuria urolithiasis. Conclusions. This study presents a global view of mRNA and miRNA expression in GHS rat kidneys, and suggests that miRNAs may be important in the regulation of hypercalciuria. The data provide valuable insights for future research, which should aim at validating the role of the genes featured here in the pathophysiology of hypercalciuria. 
1 Integrative microRNA-gene expression network analysis ingenetic 2 hypercalciuricstone-forming ratkidney

3

4 Yuchao Lu, Baolong Qin, Henglong Hu, Jiaqiao Zhang, Yufeng Wang, Qing Wang,

5 Shaogang Wang*

6

7 Department of Urology, Tongji Hospital, Tongji Medical College, Huazhong University of

8 Science and Technology, Wuhan, P.R.China

*Correspondence

Shaogang WANG, Department of Urology, Tongji Hospital, Tongji Medical College, Huazhong

University of Science and Technology, No. 1095 Jiefang Avenue, Wuhan, China.

E-mail: sgwangtjm@163.com,sgwangtjm@126.com

Tel \& Fax: +86-27-83663460

Running title: MiRNA-mRNA network in GHS rat kidney 


\section{ABSTRACT}

23

24

Background. MicroRNAs (miRNAs) influence a variety of biological functions by regulating gene expression post-transcriptionally. Aberrant miRNA expression has been associated with many human diseases. Urolithiasis is a common disease, and idiopathic hypercalciuria (IH) is an important risk factor for calcium urolithiasis. However, miRNA expression patterns and their biological functions in urolithiasis remain unknown.

Methods and Results. A multi-step approach combining microarray miRNA and mRNA expression profile and bioinformatics analysis was adopted to analyze dysregulated miRNAs and genes in genetic hypercalciuric stone-forming (GHS) rat kidneys, using normal SpragueDawley (SD) rats as controls. We identified 2418 mRNAs and 19 miRNAs as significantly differentially expressed, over 700 Gene Ontology (GO) terms and 83 KEGG pathways that were significantly enriched in GHS rats. In addition, we constructed an miRNA-gene network that suggested that rno-miR-674-5p, rno-miR-672-5p, rno-miR-138-5p and rno-miR-21-3p may play important roles in the regulatory network. Furthermore, signal-net analysis suggested that NFkappa B likely plays a crucial role in hypercalciuria urolithiasis.

Conclusions. This study presents a global view of mRNA and miRNA expression in GHS rat kidneys, and suggests that miRNAs may be important in the regulation of hypercalciuria. The data provide valuable insights for future research, which should aim at validating the role of the genes featured here in the pathophysiology of hypercalciuria. 


\section{INTRODUCTION}

43 Kidney stones are commonly found in children and adults (Coe et al. 2005), and can

44 becaused by multiple factors. Idiopathic hypercalciuria (IH) is an important risk factor forcalcium urolithiasis (Worcester et al. 2013; Yoon et al. 2013). Patients with IH have normal serum $\mathrm{Ca}^{2+}$, and increased urinary calcium excretion. But the pathophysiological process and molecular mechanism of IH are still unclear. The genetic hypercalciuric stone-forming (GHS) rat, has many pathophysiological characteristics identical to that of IH patients, such as normal serum $\mathrm{Ca}^{2+}$, hypercalciuria, elevated intestinal $\mathrm{Ca}^{2+}$ resorption and a tendency to lose calcium from the bone(Frick et al. 2013; Frick et al. 2015), which is regarded as an ideal animal model of calcium urolithiasis.

MicroRNAs (miRNAs) are a group of small, non-coding RNAs that regulate protein-coding gene function at the post-transcriptional level by binding to complementary sites on target mRNAs in the 3'UTR (Ambros 2004). Meanwhile, miRNAs have been shown to regulate a wide range of biological processes including cell growth, metabolism, differentiation, proliferation and apoptosis, which have important implications in diseases processes (Ambros 2001). of GHS and normal Sprague-Dawley (SD) rat kidneys. A subset of differentially expressed genes 
63

64 65 kidney.

66

67

68

69

6

was validated by qPCR in 12 pairs of kidneys. Bioinformatic analysis was further performed to construct an integrative regulatory network of altered miRNA-mRNA transcriptsin GHS rat

\section{MATERIALS AND METHODS}

\section{Animals}

The colony of GHS rats were created by selective breeding of male and female SpragueDawley (SD) rats with the highest 24-hour urine calcium excretion as previously described (He et al. 2015). By the 28th generation, GHS rats stably excreted significantly higher levels of urine calcium than wild-type normal SD rats. Normal SD rats were purchased from the Experimental Animal Center, Tongji Medical College, Huazhong University of Science and Technology, China. Twelve GHS rats with a body weight of 250-280 g were used for this study. A total of 12 normal SD rats were matched with GHS rats with respect to body weight and age, and served as control rats. All rats were fed $13 \mathrm{~g}$ /day of a normal calcium diet $(1.2 \%$ calcium, $0.65 \%$ phosphorus, $0.43 \%$ chloride, $0.4 \%$ sodium, and $0.24 \%$ magnesium per gram of food). All animal procedures were approved by the Ethical Committee of Tongji Hospital, Tongji Medical College, Huazhong University of Science and Technology (No. TJ-A20141211). All surgeries were performed under sodium pentobarbital anesthesia.

\section{Urine and serum calcium and phosphorus determination}

Two successive 24-hour urine samples were collected before rats were killed, then blood samples were taken after rats were killed. Urine calcium, serum calcium, and phosphorus were 
84

85

86

87

88

measured using an Abbott Aeroset AutoAnalyzer (Abbott Diagnostics, Chicago, IL, USA).

\section{RNA extraction}

Total RNA was extracted from kidney tissue using RNeasy Fibrous Tissue kit (Qiagen, Dusseldorf, Germany) according to the manufacturer's protocol. RNA purity and concentration were assessed by NanoDrop ND-2000 spectrophotometer (Thermo Fisher Scientific Inc., Waltham, MA, USA) and electrophoresis of RNA on agarose gel containing formaldehyde was used to evaluate the integrity of RNA.

\section{Microarray}

Three GHS rats and three SD rats were randomly selected for microarray analysis. The Affymetrix GeneChip miRNA 4.0 Array and Affymetrix Gene 1.0 Arrayfor rats were used to compare miRNA and mRNA expression profiles, respectively, in GHS and control rat kidneys. Microarray analysis was performed by GMINIX Informatics Ltd. Co, Shanghai, China. The data have been deposited in the NCBI Gene Expression Omnibus and are accessible through GEO Series accession number GSE75543.

\section{Strategy}

As shown in Fig. 1, we used a multi-step strategy to identify genes dysregulated in GHS rats relative to the control group. First, significantly differentially expressed mRNA and miRNA were identified using a random variance model (RVM) corrective analysis of variance (ANOVA)(Wright and Simon 2003). Second, the miRanda database was used to predict putative miRNA targets, and the overlap between these target genes and differentially expressed mRNAs was established. Third, these intersecting genes were classified according to their biological 
105

106

107

108

109

110

111

112

113

114

115

116

117

118

119

120

121

122

123

124

125

functions using the Gene Ontology System. Similarly, pathway analysis was used to identify affected KEGG pathways. Fourth, genes that were present in both the enriched GO terms and significant KEGG pathways were used to construct a miRNA-gene network (Joung et al. 2007;

Shalgi et al. 2007) and signal-net (Spirin and Mirny 2003; Zhang and Wiemann 2009). The center of the network is represented by a degree, which indicates the predicted interaction of a given miRNA with its target genes. All of the data mentioned above were analyzed by GCBI working platform (GMINIX Informatics Ltd. Co, Shanghai, P. R. China), and the principles and methodologies of data analysis were described in supplemental FileS1.Finally, a subset of the predicted miRNA-mRNA pairs was selected for validation by qRT-PCR in an extended cohort of kidney tissues.

\section{Real-time RT-PCR}

For mRNA, total RNA was extracted and 500 ng of RNA was used for cDNA synthesis using the Takara reverse transcription kit (Takara, Dalian, China) according to the manufacturer's instructions. PCR was conducted using SYBR Premix Ex Taq (Takara, Dalian, China) according to the manufacturer's instructions on an Mx3000P system (Agilent Stratagene, Santa Clara, CA, USA). The primers were chemically synthesized by Tsingke, Wuhan, China and are listed in TableS1. The All-in-One ${ }^{\mathrm{TM}}$ miRNA qRT-PCR Detection Kit (GeneCopoeia, Guangzhou, China) was used for both cDNA synthesis and quantitative detection using miRNA specific primers (GeneCopoeia, Guangzhou, China). GAPDH and U6 were used as internal controls to determine the relative expression of target mRNA and miRNA. All reactions were performed in triplicate.

\section{Statistical analysis}


126 Continuous variables were expressed as means \pm standard deviation. For each triplicate of 127 microarray data the geometric mean was used. Student's t-test was applied for comparisons 128 between two groups, and ANOVA for comparisons between more than two groups. A P-value of $129<0.05$ was considered statistically significant.

\section{RESULTS}

Serum calcium and phosphoruslevels, and urine calcium excretion

Serum calcium and phosphorus levels were not significantly altered in GHS compared with identified as significantly differentially expressed in GHS rats $(\mathrm{P}<0.05)$ including 1057 upregulated genes and 1361 downregulated genes (TableS3). 


\section{miRNA target gene prediction}

Target mRNAs for differentially expressed miRNAs were predicted using the miRanda database. Since miRNAs negatively regulate gene expression, upregulated miRNAs result in downregulated target mRNAs, and vice versa. A total of 29164 miRNA-mRNA pairs (based on 19 dysregulated miRNAs and their 10521 target mRNAs) were predicted (TableS4).

\section{Integrated analysis of dysregulated miRNAs and mRNAs}

The set of intersecting mRNAs between the predicted target mRNAs and differentially expressed mRNAs were selected (TableS5) and those that were negatively correlated with their predicted miRNA matches were used for downstream GOanalysis and KEGG pathway analysis.

There were 417 upregulated and 286downregulated GO terms $(\mathrm{P}<0.05)$, with the most significant GO terms including negative regulation of apoptotic process (GO:0043066), response to lipopolysaccharide (GO:0032496) and inflammatory response (GO:0006954). Table 2 shows the top 15 up- and downregulated GO terms, with further detail in Tables S6 and S7.

According to KEGG pathway analysis, 93 KEGG pathways were significantly enriched, of which 75 were upregulated and 18 downregulated at $\mathrm{P}<0.05$ (Tables S8 and S9). The most highly enriched pathways included the Pantothenate and CoA biosynthesis and synthesis and degradation of ketone bodies pathways. The top up- and downregulated pathways are shown in Fig. 4.The darker bars indicate pathways that reported to be related to urolithasis in previous studies.

Next, to illustrate the role of key miRNAs in the regulation of kidney mRNAs in GHS rats, a miRNA-mRNA-Network was built based on the subset of significantly differentially expressed 
168 169

mRNAs that were also members of significantly enriched GO terms and KEGG pathways (TableS10, Fig. 5). In total, 223 mRNAs and 19 miRNAs were included in the network, where box nodes represent miRNAs, and circular nodes represent mRNAs. The degree of connectivity, which represents the number of genes regulated by a given miRNA, is indicated by the size of the node with a higher degree of connectivity represented by larger nodes (TableS11). Rno-miR674-5p, rno-miR-672-5p, rno-miR-138-5p, and rno-miR-21-3p had high degrees of connectivity and may play crucial roles in this regulatory network. Meanwhile, Sema5a, Lpin2, Gcnt1, Masp1, Olr1 and Traf3 were the most common miRNAtargets. Table 3 presented a subset of significantly regulated miRNA-mRNA hybrids.

Moreover, to investigate the regulatory relationships between these genes and their potential role in hypercalciuria, we performed a signal-net analysis based on significantly regulated KEGG pathways (Fig.6, Table S12). Signal-net analysis has shown that NF-kappa B signal pathway, including the members of NF-kappa B1, RelA and NF-kappa B2 et al, might play a core role in the gene regulatory network. And NF-kappa B1 and RelA had the highest degrees of connectivity of 19 and 18, respectively.

\section{Real-time quantitative PCR validation}

To validate the reliability of our microarray-based results, 11 miRNAs and eight target mRNAs were selected for validation by qRT-PCR in 12 pairs of matched GHS / normal SD rat kidneys (TableS13). As demonstrated in Fig.7-A, rno-miR-184, rno-miR-484 and rno-miR-1381-3pwere the most significantly dysregulated miRNAs in GHS rats, and the results were 
189 190

comparable with our microarray data. Moreover, the expression levels of the seven mRNAs measured by qPCR (Gcnt1, Lpin2, Olr1, Sema5a, NfkB1, Rela and VDR) coincided exactly with our microarray data except for CaSR (Fig. 7-B).

These results demonstrate a strong consistency between the microarray- and qRT-PCR-based results.

\section{DISCUSSION}

IH typically manifests as hypercalciuria with normal serum $\mathrm{Ca}^{2+}$, increased intestinal $\mathrm{Ca}^{2+}$ absorption, bone resorption and decreased bone mineral density in addition to decreased renal tubule $\mathrm{Ca}^{2+}$ reabsorption (Yoon et al. 2013).Thus, $\mathrm{IH}$ is one of the most important risk factors for calcium urolithiasis. However, the pathogenesis of $\mathrm{IH}$ is not yet fully understood. GHS rats exhibit many features of human $\mathrm{IH}$ including increased intestinal $\mathrm{Ca}^{2+}$ absorption, increased bone resorption, decreased renal tubule $\mathrm{Ca}^{2+}$ reabsorption and high levels of vitamin $\mathrm{D}$ receptor (VDR) protein in $\mathrm{Ca}^{2+}$-transporting organs (Frick et al. 2013; Frick et al. 2015). The research of Tsuruoka et al. (Tsuruoka et al. 1997) strongly suggests that decreased tubular $\mathrm{Ca}^{2+}$ reabsorption plays a key role in hypercalciuria. In general, GHS rats represent an ideal animal model for idiopathic hypercalciuria urolithiasis.

MicroRNAs regulate various disease processes by inhibiting the expression of their target mRNAs. Understanding the relevance of miRNA and mRNA expression patterns in GHS rat kidneys is important to better elucidate the relationship between pathophysiological process and genes. In the present study, we used bioinformatics methods to determine the potential role of 
210 differentially expressed miRNAs and mRNAs in GHS rat kidneys, and identified specific

211 miRNAs and possible negative regulative mRNAs that may affect the development of

212 hypercalciuria.

213 We used a multi-step approach to identify mRNA targets of dysregulated miRNAs in GHS 214 rat kidneys whereby we first identified mRNAs $(\mathrm{N}=2418)$ and miRNAs $(\mathrm{N}=19)$ that were 215 significant differentially expressed between GHS and control SD rats; next, potential miRNAmRNA pairs were predicted for the 19 miRNAs using miRanda, resulting in29164 miRNA217 mRNA pairs; the intersecting set of predicted target mRNAs and differentially expressed mRNAs were then selected for further analysis.

219 We found a significant enrichment in over $700 \mathrm{GO}$ terms, including inflammatory response 220 (GO: 0006954) and response to hypoxia (GO: 0001666). Interestingly, local hypoxic conditions and inflammatory injuries have been linked to the initiation of urolithiasis (Cao et al. 2006), whereas antioxidants protect renal tubular cells from cellular injury and decrease the formation of calcium oxalate stones (Itoh et al. 2005). Moreover, the GO term response to calcium ion (GO: 0051592) was enriched (based on differentially expressed genes such asIL-6 and VDR). Increased levels of VDR protein in the kidney are regarded as a common feature in both GHS rats and IH individuals (Frick et al. 2015), and VDR intimately affects urolithiasis formation

227 (Arcidiacono et al. 2014; Zhang et al. 2013). Glybochko et al. found a significant increase in serum IL-6 in nephrolithiasis patients compared with healthy individuals (Glybochko et al. 2010), and Hasna et al. showed that IL-6 was significantly increased in patients with diabetes mellitus and urolithiasis compared with patients with diabetes mellitus alone (Hasna et al. 2015). In 
231 addition, skeletal system development (GO: 0001501) and skeletal system morphogenesis (GO:

232 0048705) were significantly increased in GHS rats. Our previous studies demonstrated the

233 occurrence of osteochondral differentiation progression in primary renal tubular epithelial cells

234 in GHS rats (He et al. 2015), which may be linked to the pathogenesis of calcium stone

235 development.

236 By identifying pathway membership of dysregulated mRNAs, we can improve our 237 understanding of underlying disease-related processes. We detected 93 enriched pathways including the MAPK signaling pathway, mineral absorption as well as the TGF-beta signaling pathway. Khan et al. reported that the p38 MAPK/JNK pathway regulates crystallization modulator production and influences plaque formation as well as calcium oxalate nephrolithiasis (Khan 2013). Our previous research also showed that calcium and TGF- $\beta$ may participate in the pathogenesis of epithelial-mesenchymal transition and lead to stone formation (He et al. 2015).

To better understand the biological processes linked to differentially expressed miRNAs and their predicted target genes, we constructed an interaction network where rno-miR-674-5p, rnomiR-672-5p, rno-miR-138-5p and rno-miR-21-3p were found to be highly connected, which means that they may play crucial roles in the regulatory network. Interestingly, Wang et al. 247 reported that miR-674-5p was able to stimulate the expression of osteogenic marker genes 248 (Wang et al. 2011); miR-138-5p is a risk factor for pancreatic cancer (Yu et al. 2015) and 249 Alzheimer disease (Lugli et al. 2015); and miR-21-3p increases resistance to cisplatin in a range of ovarian cell lines (Pink et al. 2015). In addition, rno-miR-484, rno-miR-138-1-3p, rno-miR201-3p and rno-miR-203a-3p are downregulated in the network, nevertheless, previous studies 
252 reported these miRNAs to be tumor-associated (Pizzini et al. 2013; Liu et al. 2015; Merhautova

253 et al. 2015; Murray et al. 2014; Yang et al. 2016; Ye et al. 2015). To our knowledge, there has

254 been very little research on the relationship between these miRNAs and urolithiasis or calcium

255 metabolism. Hu et al. reported serum and urinary levels of miR-155 were significantly elevated

256 in patients with nephrolithiasis, and miR-155 might influence pathophysiology of nephrolithiasis

257 via regulating inflammatory cytokines expression (Hu et al. 2014). However, the expressions of

miR-155were not significantly different between the two groups of our present study. Regarding

mRNAs in the constructed network, Sema5a, which belongs to the semaphorin gene family, had

the highest degree of connectivity of 13. It has previously been reported that Sema5a increases

cell proliferation and metastasis and suppresses tumor formation (Lu et al. 2010; Sadanandam et

al. 2012). In addition, Ding $\mathrm{H}$ et al. suggested that Sema5a is a risk factor for Parkinson's disease

(Ding et al. 2008). Lpin2, which is associated with fatty acid, triacylglycerol, and ketone body

metabolism (Chen et al. 2015), also had a high degree of connectivity of 9. Olr1, a crucial

regulator of lipid metabolism (Tejedor et al. 2015), had a degree of connectivity of 8. Increasing

numbers of studies are reporting a strong correlation between obesity/dyslipidemia and kidney

stones (De SK et al. 2014; Fujimura et al. 2014). However, the mechanism whereby obesity and

kidney stone disease are linked is still unknown. Recent research has indicated that obesity may

increase reactive oxygen species and oxidative stress, which would influence the interaction

between calcium oxalate/calcium phosphate crystals and renal epithelial cells (Khan 2012).

The NF-kappa B signaling pathway was also significantly enriched in GHS rats, in addition, 
273 regulatory network including the members of NF-kappa B1, NF-kappa B2 and RelA. NF-kappa

$274 \mathrm{~B}$ is a transcription regulator that is activated by various intra- and extra-cellular stimuli such as 275 cytokines, oxidant-free radicals, and bacterial or viral products (Hoesel and Schmid 2013).

276 Activated NF-kappa B translocates into the nucleus and stimulates the expression of genes

277 involved in a wide variety of biological functions. Menon et al. (Menon et al. 2014) found that

278 the receptor activator of NF-kappa B ligand mediates bone resorption in IH, while Tozawa et al.

279 (Tozawa et al. 2008) reported that oxalate induced OPN expression by activating NF-kappa B in renal tubular cells. But there have been few researches focus on the function of NF-kappa B in

281 the formation of idiopathic hypercalciuria urolithiasis. Furthermore, abnormal fat metabolism would also activate the NF-kappa B signaling pathway (Yang et al. 2015), which might contribute to the increased risk of urolithiasis in obesity populations. However, the molecular mechanism of NF-kappa B in IH desease are need further explore, which may assist in improving the clinical diagnosis and treatment of patients with $\mathrm{IH}$.

CONCLUSIONS

Here, we successfully identify rat hypercalciuria-related miRNAs and their target mRNAs. This comprehensive analysis will provide valuable insights for future research, which should aim at confirming the role of these genes in the pathophysiology of hypercalciuria stone desease.

More further work needs to be done to get the whole picture right in the disease context. To the best of our knowledge, this is the first study to focus on the role of miRNA-mRNA interactions in hypercalciuria urolithiasis. 
295

296

297

298

299

300

301

302

303

304

305

306

307

308

309

310

311

312

313

314

315

316

317

318

319

320

321

322

323

324

325

326

327

328

329

\section{ACKNOWLEDGMENTS}

We thank GMINIX Informatics Ltd. Co (Shanghai, P. R. China) for their technical assistance with bioinformatics analysis.

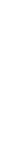

Frick KK, Krieger NS, and Bushinsky DA. 2015. Modeling hypercalciuria in the genetic hypercalciuric stoneforming rat. Curr Opin Nephrol Hypertens 24(4):336-344.

Fujimura M, Sakamoto S, Sekita N, Mikami K, Ichikawa T, and Akakura K. 2014. Visceral fat accumulation is a risk factor for urinary stone. INT J UROL 21(11):1184-1185.

Glybochko PV, Svistunov AA, Rossolovskii AN, Ponukalin AN, Zakharova NB, Bliumberg BI, and Berezinets OL. 2010. [Endothelial dysfunction in urolithiasis patients]. Urologiia(3):3-6.

Hasna A, Meiyappan K, Periyasam SG, Kalyaperumal M, Bobby Z, and Subramaniam AV. 2015. Is urolithiasis associated with increased levels of high sensitivity C-reactive protein and interleukin-6 in diabetic patients? J Clin Diagn Res 9(3):C1-C3.

He D, Lu Y, Hu H, Zhang J, Qin B, Wang Y, Xing S, Xi Q, and Wang S. 2015. The Wnt11 Signaling Pathway in Potential Cellular EMT and Osteochondral Differentiation Progression in Nephrolithiasis Formation. INT J MOL SCI 16(7):16313-16329. 
Hoesel B, and Schmid JA. 2013. The complexity of NF-kappaB signaling in inflammation and cancer. MOL CANCER 12:86.

Hu YY, Dong WD, Xu YF, Yao XD, Peng B, Liu M, and Zheng JH. 2014. Elevated levels of miR-155 in blood and urine from patients with nephrolithiasis. BIOMED RES INT 2014:295651.

Itoh Y, Yasui T, Okada A, Tozawa K, Hayashi Y, and Kohri K. 2005. Examination of the anti-oxidative effect in renal tubular cells and apoptosis by oxidative stress. Urol Res 33(4):261-266.

Joung JG, Hwang KB, Nam JW, Kim SJ, and Zhang BT. 2007. Discovery of microRNA-mRNA modules via population-based probabilistic learning. BIOINFORMATICS 23(9):1141-1147.

Khan SR. 2012. Is oxidative stress, a link between nephrolithiasis and obesity, hypertension, diabetes, chronic kidney disease, metabolic syndrome? Urol Res 40(2):95-112.

Khan SR. 2013. Reactive oxygen species as the molecular modulators of calcium oxalate kidney stone formation: evidence from clinical and experimental investigations. J Urol 189(3):803-811.

Liu Y, Dong Z, Liang J, Guo Y, Guo X, Shen S, Kuang G, and Guo W. 2015. Methylation-mediated repression of potential tumor suppressor miR-203a and miR-203b contributes to esophageal squamous cell carcinoma development. Tumour Biol.

Lu TP, Tsai MH, Lee JM, Hsu CP, Chen PC, Lin CW, Shih JY, Yang PC, Hsiao CK, and Lai LC and others. 2010. Identification of a novel biomarker, SEMA5A, for non-small cell lung carcinoma in nonsmoking women. Cancer Epidemiol Biomarkers Prev 19(10):2590-2597.

Lugli G, Cohen AM, Bennett DA, Shah RC, Fields CJ, Hernandez AG, and Smalheiser NR. 2015. Plasma Exosomal miRNAs in Persons with and without Alzheimer Disease: Altered Expression and Prospects for Biomarkers. PLOS ONE 10(10):e139233.

Menon VB, Moyses RM, Gomes SA, de Carvalho AB, Jorgetti V, and Heilberg IP. 2014. Expression of fibroblast growth factor 23, vitamin D receptor, and sclerostin in bone tissue from hypercalciuric stone formers. Clin J Am Soc Nephrol 9(7):1263-1270.

Merhautova J, Hezova R, Poprach A, Kovarikova A, Radova L, Svoboda M, Vyzula R, Demlova R, and Slaby O. 2015. miR-155 and miR-484 Are Associated with Time to Progression in Metastatic Renal Cell Carcinoma Treated with Sunitinib. BIOMED RES INT 2015:941980.

Murray MJ, Bailey S, Raby KL, Saini HK, de Kock L, Burke GA, Foulkes WD, Enright AJ, Coleman N, and Tischkowitz M. 2014. Serum levels of mature microRNAs in DICER1-mutated pleuropulmonary blastoma. Oncogenesis 3:e87.

Pink RC, Samuel P, Massa D, Caley DP, Brooks SA, and Carter DR. 2015. The passenger strand, miR-21-3p, plays a role in mediating cisplatin resistance in ovarian cancer cells. GYNECOL ONCOL 137(1):143-151.

Pizzini S, Bisognin A, Mandruzzato S, Biasiolo M, Facciolli A, Perilli L, Rossi E, Esposito G, Rugge M, and Pilati $\mathrm{P}$ and others. 2013. Impact of microRNAs on regulatory networks and pathways in human colorectal carcinogenesis and development of metastasis. BMC GENOMICS 14:589.

Sadanandam A, Sidhu SS, Wullschleger S, Singh S, Varney ML, Yang CS, Ashour AE, Batra SK, and Singh RK. 2012. Secreted semaphorin 5A suppressed pancreatic tumour burden but increased metastasis and endothelial cell proliferation. Br J Cancer 107(3):501-507.

Shalgi R, Lieber D, Oren M, and Pilpel Y. 2007. Global and local architecture of the mammalian microRNAtranscription factor regulatory network. PLOS COMPUT BIOL 3(7):e131.

Spirin V, and Mirny LA. 2003. Protein complexes and functional modules in molecular networks. Proc Natl Acad 
371

372

373

374

375

376

377

378

379

380

381

382

383

384

385

386

387

388

389

390

391

392

393

394

395

396

397

398

399

400

401

Sci U S A 100(21):12123-12128.

Tejedor JR, Tilgner H, Iannone C, Guigo R, and Valcarcel J. 2015. Role of six single nucleotide polymorphisms, risk factors in coronary disease, in OLR1 alternative splicing. RNA 21(6):1187-1202.

Tozawa K, Yasui T, Okada A, Hirose M, Hamamoto S, Itoh Y, and Kohri K. 2008. NF-kappaB activation in renal tubular epithelial cells by oxalate stimulation. INT J UROL 15(10):924-928.

Tsuruoka S, Bushinsky DA, and Schwartz GJ. 1997. Defective renal calcium reabsorption in genetic hypercalciuric rats. KIDNEY INT 51(5):1540-1547.

Wang Y, Jiang XL, Yang SC, Lin X, He Y, Yan C, Wu L, Chen GQ, Wang ZY, and Wu Q. 2011. MicroRNAs in the regulation of interfacial behaviors of MSCs cultured on microgrooved surface pattern. BIOMATERIALS 32(35):9207-9217.

Worcester EM, Bergsland KJ, Gillen DL, and Coe FL. 2013. Evidence for increased renal tubule and parathyroid gland sensitivity to serum calcium in human idiopathic hypercalciuria. Am J Physiol Renal Physiol 305(6):F853F860.

Wright GW, and Simon RM. 2003. A random variance model for detection of differential gene expression in small microarray experiments. BIOINFORMATICS 19(18):2448-2455.

Yang SJ, Choi JM, Park SE, Rhee EJ, Lee WY, Oh KW, Park SW, and Park CY. 2015. Preventive effects of bitter melon (Momordica charantia) against insulin resistance and diabetes are associated with the inhibition of NFkappaB and JNK pathways in high-fat-fed OLETF rats. J NUTR BIOCHEM 26(3):234-240.

Yang Y, Lin X, Lu X, Luo G, Zeng T, Tang J, Jiang F, Li L, Cui X, and Huang W and others. 2016. InterferonmicroRNA signalling drives liver precancerous lesion formation and hepatocarcinogenesis. GUT.

Ye FG, Song CG, Cao ZG, Xia C, Chen DN, Chen L, Li S, Qiao F, Ling H, and Yao L and others. 2015. Cytidine Deaminase Axis Modulated by miR-484 Differentially Regulates Cell Proliferation and Chemoresistance in Breast Cancer. CANCER RES 75(7):1504-1515.

Yoon V, Adams-Huet B, Sakhaee K, and Maalouf NM. 2013. Hyperinsulinemia and urinary calcium excretion in calcium stone formers with idiopathic hypercalciuria. J Clin Endocrinol Metab 98(6):2589-2594.

Yu C, Wang M, Li Z, Xiao J, Peng F, Guo X, Deng Y, Jiang J, and Sun C. 2015. MicroRNA-138-5p regulates pancreatic cancer cell growth through targeting FOXC1. Cell Oncol (Dordr) 38(3):173-181.

Zhang JD, and Wiemann S. 2009. KEGGgraph: a graph approach to KEGG PATHWAY in R and bioconductor. BIOINFORMATICS 25(11):1470-1471.

Zhang P, Nie W, and Jiang H. 2013. Effects of vitamin D receptor polymorphisms on urolithiasis risk: a metaanalysis. BMC MED GENET 14:104. 


\section{Table $\mathbf{1}$ (on next page)}

19 dysregulated miRNAs

The significantly dysregulated miRNAs between GHS and SD rats 
1 Table 1. The significantly dysregulated miRNAs between GHS and SD rats.

\begin{tabular}{|c|c|c|c|}
\hline miRNA & P-value & Fold-change & style \\
\hline rno-miR-184 & 0.00069 & 29.41 & up \\
\hline rno-miR-21-3p & 0.001198 & 4.17 & up \\
\hline rno-miR-672-5p & 0.047372 & 2.70 & up \\
\hline rno-miR-6324 & 0.018993 & 2.50 & up \\
\hline rno-miR-154-5p & 0.028899 & 2.13 & up \\
\hline rno-miR-770-3p & 0.040784 & 1.64 & up \\
\hline rno-miR-674-5p & 0.003039 & 1.59 & up \\
\hline rno-miR-376a-3p & 0.02666 & 1.52 & up \\
\hline rno-miR-99b-3p & 0.049668 & 1.25 & up \\
\hline rno-miR-146a-5p & 0.040208 & 1.18 & up \\
\hline rno-miR-203b-3p & 0.018288 & 0.65 & down \\
\hline rno-miR-20b-3p & 0.032176 & 0.64 & down \\
\hline rno-miR-206-3p & 0.006578 & 0.45 & down \\
\hline rno-miR-196c-3p & 0.028658 & 0.38 & down \\
\hline rno-miR-138-5p & 0.018426 & 0.32 & down \\
\hline rno-miR-203a-3p & 0.03408 & 0.30 & down \\
\hline rno-miR-201-3p & 0.03048 & 0.25 & down \\
\hline rno-miR-138-1-3p & 0.00485 & 0.11 & down \\
\hline
\end{tabular}




\begin{tabular}{llll}
\hline rno-miR-484 & 0.004176 & 0.08 & down \\
\hline
\end{tabular}

2 
Table 2 (on next page)

GO-dysregulated

The top 15 up- and downregulated GO terms 


\section{Table 2. MicroRNA targets significant GO}

\begin{tabular}{|c|c|c|c|}
\hline GO-ID & GO-name & enrichment & P-value \\
\hline \multicolumn{4}{|c|}{ Upregulated GOs by GO analysis } \\
\hline GO:0043066 & negative regulation of apoptotic process & 5.274431709 & $5.53034 \mathrm{E}-12$ \\
\hline GO:0032496 & response to lipopolysaccharide & 8.108054973 & $1.51933 \mathrm{E}-11$ \\
\hline GO:0006954 & inflammatory response & 8.099023444 & $2.11218 \mathrm{E}-10$ \\
\hline GO:0007165 & signal transduction & 5.49328365 & $2.53815 \mathrm{E}-10$ \\
\hline GO:0071347 & cellular response to interleukin-1 & 16.88289078 & $1.09298 \mathrm{E}-08$ \\
\hline \multirow[t]{2}{*}{ GO:0045944 } & positive regulation of transcription from & 3.652056561 & $1.27896 \mathrm{E}-08$ \\
\hline & RNA polymerase II promoter & & \\
\hline GO:0014070 & response to organic cyclic compound & 6.26379716 & $3.29652 \mathrm{E}-08$ \\
\hline GO:0008285 & negative regulation of cell proliferation & 5.262199724 & $5.94266 \mathrm{E}-08$ \\
\hline GO:0033590 & response to cobalamin & 90.04208417 & $6.01314 \mathrm{E}-08$ \\
\hline GO:0042493 & response to drug & 4.287718294 & $6.34324 \mathrm{E}-08$ \\
\hline GO:0006468 & protein phosphorylation & 4.846457788 & $8.88401 \mathrm{E}-08$ \\
\hline GO:0042542 & response to hydrogen peroxide & 12.4673655 & $1.76279 \mathrm{E}-07$ \\
\hline GO:0009617 & response to bacterium & 19.09983604 & $2.66 \mathrm{E}-07$ \\
\hline GO:0001889 & liver development & 8.612721094 & $2.86336 \mathrm{E}-07$ \\
\hline GO:0001666 & response to hypoxia & 5.58112092 & 4.40694E-07 \\
\hline
\end{tabular}




\begin{tabular}{|c|c|c|c|}
\hline GO:0045893 & positive regulation of transcription, & 3.77523152 & $3.05206 \mathrm{E}-07$ \\
\hline \multirow{3}{*}{ GO:0045892 } & DNA-dependent & \multirow{3}{*}{4.062477396} & \multirow{3}{*}{$3.32485 \mathrm{E}-07$} \\
\hline & negative regulation of transcription, & & \\
\hline & DNA-dependent & & \\
\hline GO:0016568 & chromatin modification & 13.26523231 & $5.79738 \mathrm{E}-07$ \\
\hline GO:0043065 & positive regulation of apoptotic process & 5.038731654 & $6.66823 \mathrm{E}-07$ \\
\hline GO:0008150 & biological process & 2.365931438 & $1.86723 \mathrm{E}-06$ \\
\hline GO:0016572 & histone phosphorylation & 46.4283131 & $3.08593 \mathrm{E}-06$ \\
\hline \multirow[t]{2}{*}{ GO:0045944 } & positive regulation of transcription from & 2.954529015 & 4.94117E-06 \\
\hline & RNA polymerase II promoter & & \\
\hline GO:0006355 & regulation of transcription, DNA-dependent & 2.982729366 & $6.6796 \mathrm{E}-06$ \\
\hline GO:0003407 & neural retina development & 36.11091019 & $1.08935 \mathrm{E}-05$ \\
\hline GO:0001525 & angiogenesis & 5.879901494 & $1.29214 \mathrm{E}-05$ \\
\hline GO:0008284 & positive regulation of cell proliferation & 3.559902873 & 3.26349E-05 \\
\hline GO:0006950 & response to stress & 5.762379285 & 4.3047E-05 \\
\hline GO:0006915 & apoptotic process & 3.631264712 & 4.68891E-05 \\
\hline GO:0007165 & signal transduction & 3.448256676 & 8.82113E-05 \\
\hline GO:0043066 & negative regulation of apoptotic process & 3.059606407 & 0.000134769 \\
\hline
\end{tabular}




\section{Table 3 (on next page)}

miRNA-mRNA hybrid

miRNAs and target mRNAs in GHS rat kidneys. 
1 Table 3. miRNAs and target mRNAs in GHS rat kidneys.

\begin{tabular}{|c|c|}
\hline miRNA & Target mRNAs \\
\hline \multirow[t]{2}{*}{ rno-miR-138-1-3p } & Cxcl16, Cyp4a1, Dnm3, Ehhadh, Hbegf, Hspa41, Inhbb, Masp1, \\
\hline & Mmp9, Nr4a3, Rprm, Sema5a, Steap2 \\
\hline \multirow[t]{4}{*}{ rno-miR-138-5p } & Aass, Adh1, Ak7, Bhmt, Calcr, Cdh2, Csf2rb, Cxc11, F3, Ggcx, \\
\hline & Gna12, Gpd1, Gsk3a, Gstt1, Hbegf, Hmgcl, Hspa41, Icam1, Inhbb, \\
\hline & Lpin2, Nr4a3, Pnpla2, Rela, Sds, Sema4b, Sema4c, Sema5a, Spry4, \\
\hline & Tmprss 13, Zfp36 \\
\hline \multirow[t]{2}{*}{ rno-miR-196c-3p } & Agpat9, Cebpb, Dusp16, Hbegf, Inhbb, Masp1, Nfkbia, Nfkbiz, Olr1, \\
\hline & Pvrl1, Rel, Sema4c, Sorbs1, Tnfrsf21 \\
\hline \multirow[t]{3}{*}{ rno-miR-201-3p } & Amt, Bcl10, Crem, Ctsd, Cubn, Ddit4, Dusp16, Entpd3, Got2, Gpx1, \\
\hline & Hbegf, I123a, Lpin2, Map2k3, Masp1, Nr4a3, Ppp2r1a, Sema5a, Sept8, \\
\hline & Tmprss 13, Vdr \\
\hline \multirow[t]{3}{*}{ rno-miR-203a-3p } & Angpt14, B3gnt5, Bcl10, Bhmt, Crem, Cxcl1, Cxcl11, Cyp1b1, Dpys, \\
\hline & Hbegf, Il2ra, Inhbb, Lpin2, Nr4a3, Olr1, Qdpr, S1pr1, Sema4g, \\
\hline & Sema5a, Serpine1, Sorbs1, Steap2, Tpm3, Vcam1, Xbp1 \\
\hline \multirow[t]{3}{*}{ rno-miR-206-3p } & Amt, Angpt4, Angpt14, Cdh2, Crem, Cyp4a1, Dnm3, Ehhadh, \\
\hline & Gadd45b, Icam1, I16, Map3k8, Mchr1, Ncoa4, Nr1h3, Nr4a3, Olr1, \\
\hline & Pex11a, Pla2g15, Sema4g, Sema5a, Sgk1, Spry4, Tpm3, Zfp36 \\
\hline rno-miR-484 & Angpt14, B4galnt1, Bcl10, Chrd, Cox4i1, Cxcl16, Ddit4, Flt3, Foxo3, \\
\hline
\end{tabular}


Gadd45b, Gcat, Gpd1, Hyal2, Mgat5, Ncoa4, Nfkbiz, P2rx7, Pex11a,

Pim1, Ppara, Rprm, Runx1, S1pr1, Scarb1, Sele, Sema4g, Sema5a, Sema6b, Sorbs1, Tnfrsf21, Tpm3

rno-miR-184

rno-miR-21-3p

rno-miR-672-5p

rno-miR-674-5p
Alg2, Dnm1, Lpcat1, Ndst1, Ocln, Prom1, Ube2n, Wwp1

Ace, Adam10, Adh6, Cacnb2, Cdc14b, Cycs, Dut, Egf, Ganc, Ggps1,

Itpr2, Mboat2, Mecom, Ocln, Plau, Pld1, Rgn, Rps6ka5, Snca, Traf3,

Whsc1

Agtr1b, Alg2, Creb312, Dnm1, Gcnt1, Ggps1, Itpr2, Lpcat1, Ndst1, Prom1, Rb12, Scp2, Sh3g12, Sin3a, Smad6, Stat1, Traf3

Adam10, Adh6, Agtr1b, Alg2, Capn1, Dgkz, Dnmt1, Ganc, Ggps1, Hdac7, Itpr2, Mavs, Mef2c, Ndufs4, Rbl1, Rbl2 
1

The multi-step strategy used in this study.

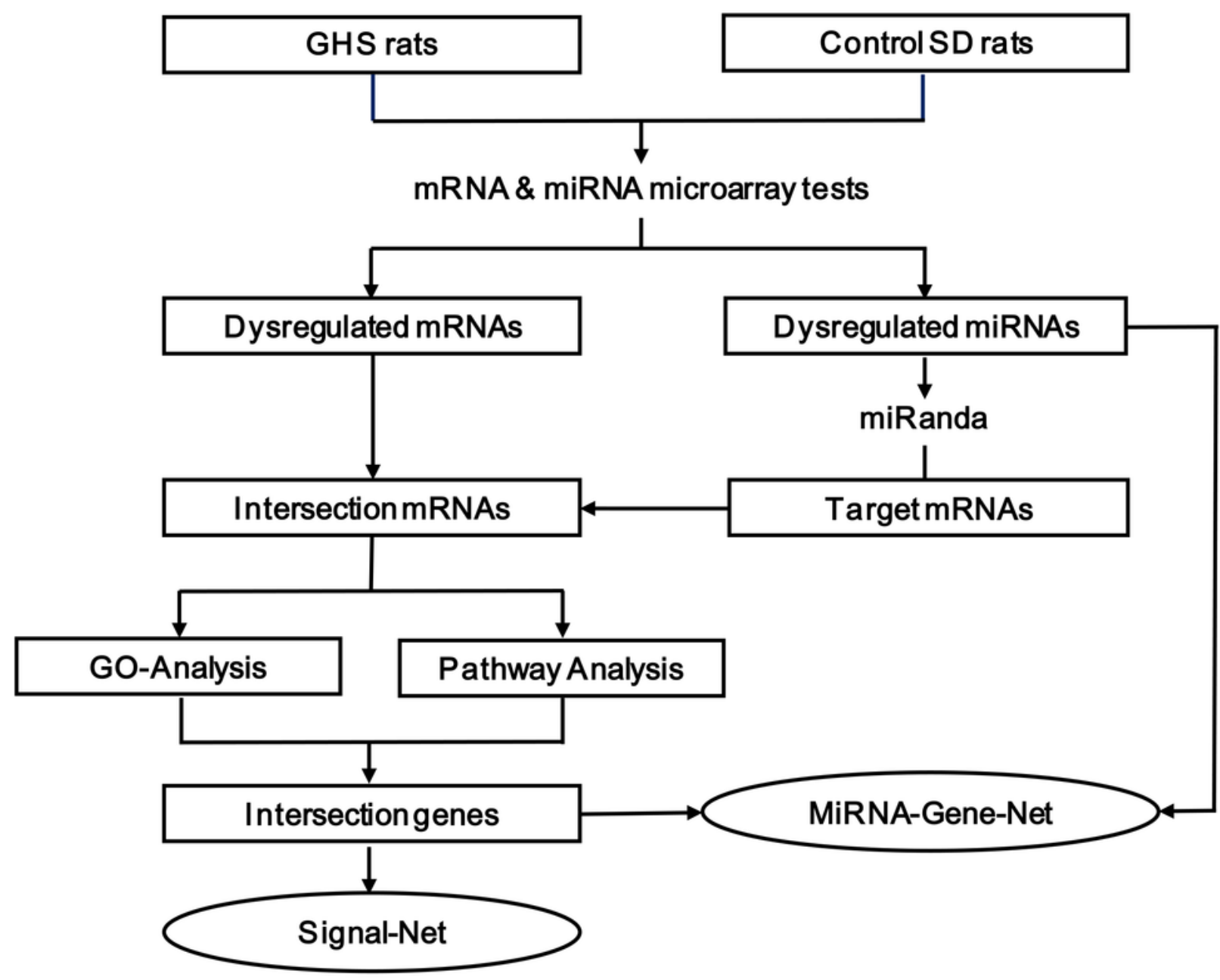


2

Serum calcium, phosphorus and urine calcium levels of GHS and normal SD rats.

The serum calcium (A) and serum phosphorus (B) levels were not significantly altered in GHS compared with SD rats. The 24-h urine calcium (C) level in GHS rats was significantly increased. Symbol $(*)$ indicates significant difference from control $(P<0.05)$.

A

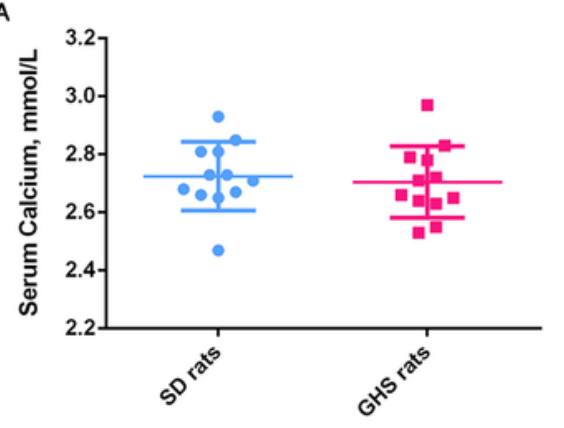

B

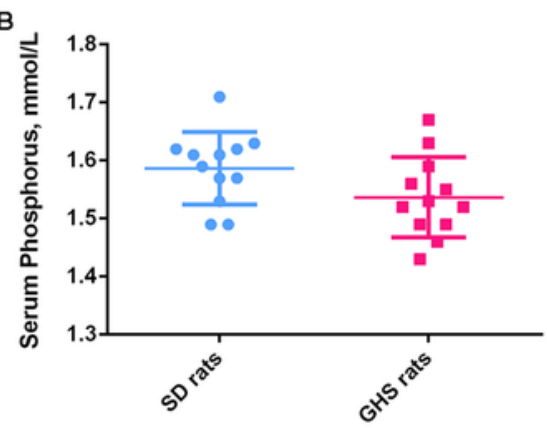

C

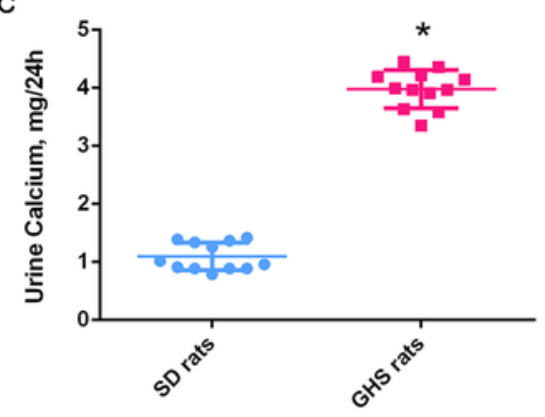


3

Histogram of signaling pathways based on the intersecting genes from predicted target mRNAs and differentially expressed mRNAs.

(A) and (B) show significant upregulated and downregulated pathways, respectively. X-axis, negative logarithm of the P-value (-LgP); Y-axis, pathway. The higher the -LgP, the lower the P-value. The darker bars indicate pathways relate to urolithasis.

A

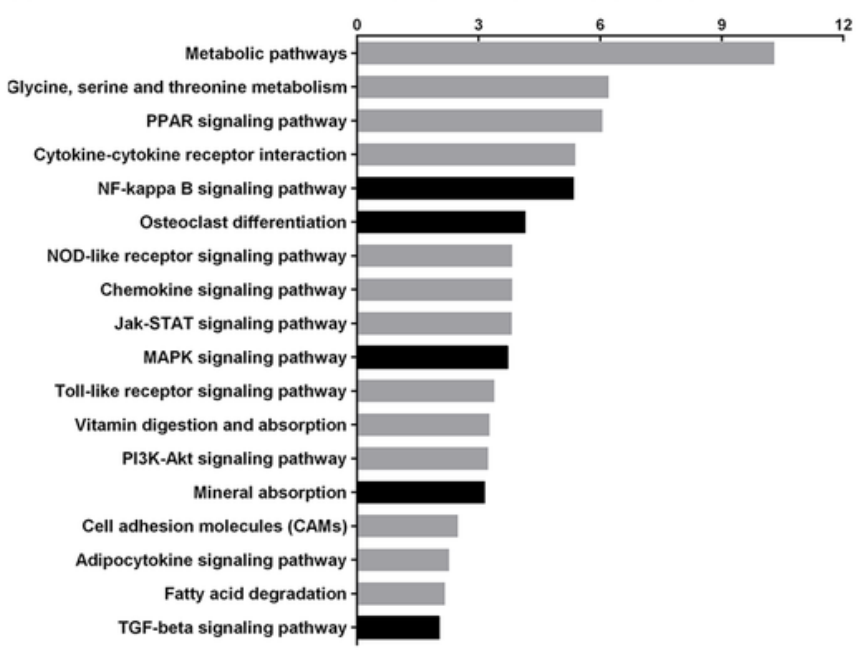

B

Downregulated pathway (- $\log \mathrm{P})$

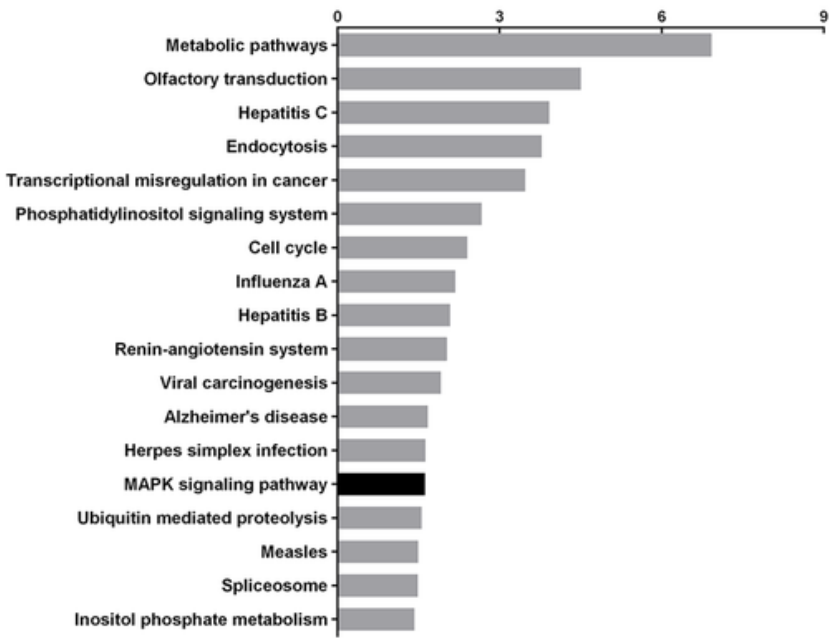


4

miRNA-gene-network.

Box nodes represent miRNAs, circular nodes represent mRNAs. Green represents

downregulation, while red represents upregulation. The higher the degree of connectivity of a gene, the larger the node within the network. In total, 223 mRNAs and 19 miRNAs were included in the network. Rno-miR-674-5p, rno-miR-672-5p, rno-miR-138-5p, and rno-miR-21$3 p$ were found to have the highest degrees of connectivity.

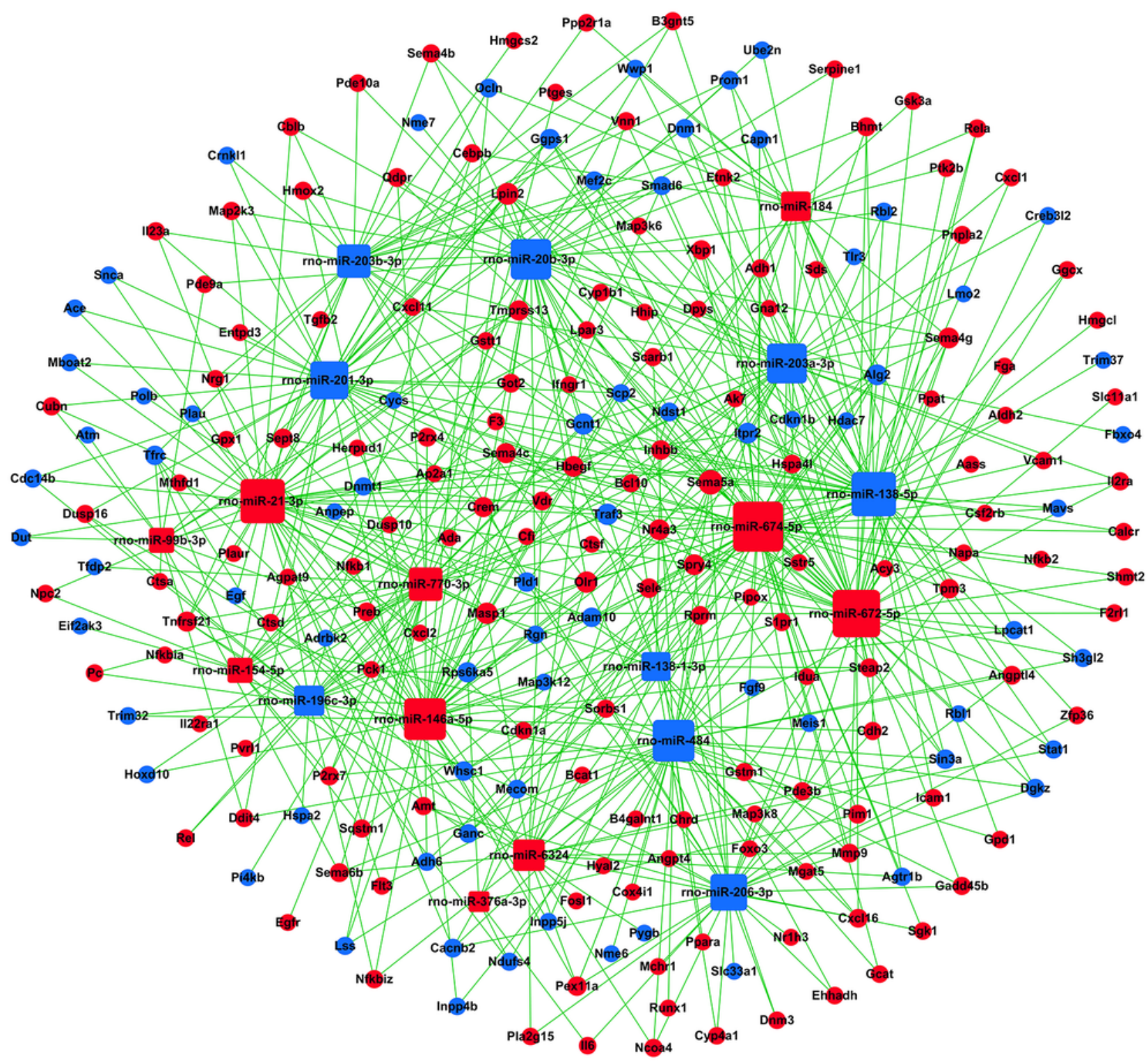




\section{5}

Signal-net.

Green, downregulation; red, upregulation; a, activation; a(ind), activation(indirect effect); a(ind)(p), activation(indirect effect)(phosphorylation); a(p), activation(phosphorylation); b, binding/association; disso, dissociation; disso(ex), dissociation(expression); disso(inh), dissociation(inhibition); ex, expression; ind(inh), indirect effect(inhibition); inh, inhibition; $\operatorname{inh}(s)$, inhibition(state change); inh(u), inhibition(ubiquitination); p, phosphorylation; $u$, ubiquitination.

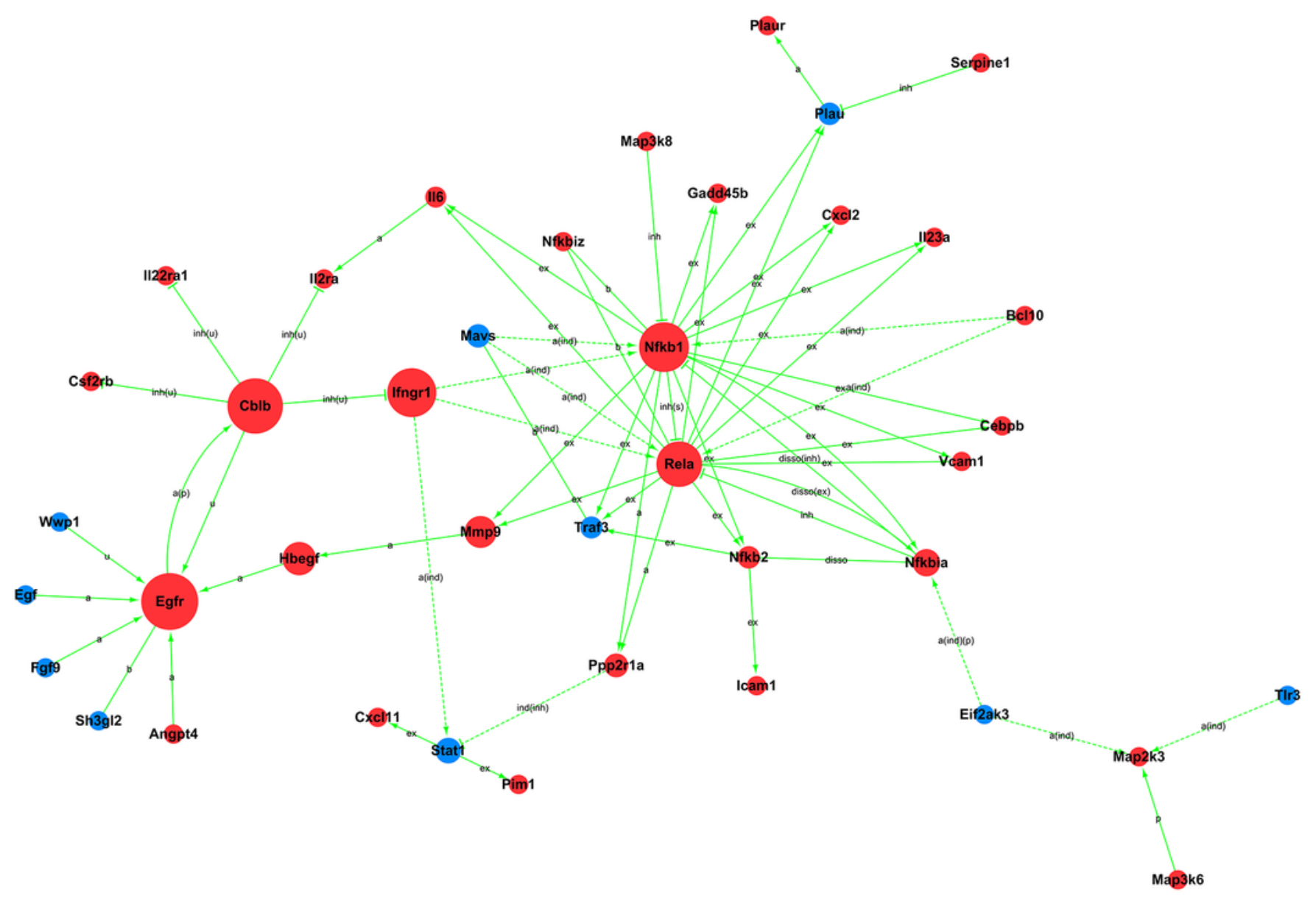


6

qRT-PCR was performed to confirm the expression of 11 selected miRNAs and eight mRNAs.

Quantitative reverse transcription PCR (qRT-PCR) validation of differentially expressed miRNAs (A) and mRNAs (B). Results are shown as mean of $\log 2$ (GHS/SD) and are presented side by side with the respective microarray results. 


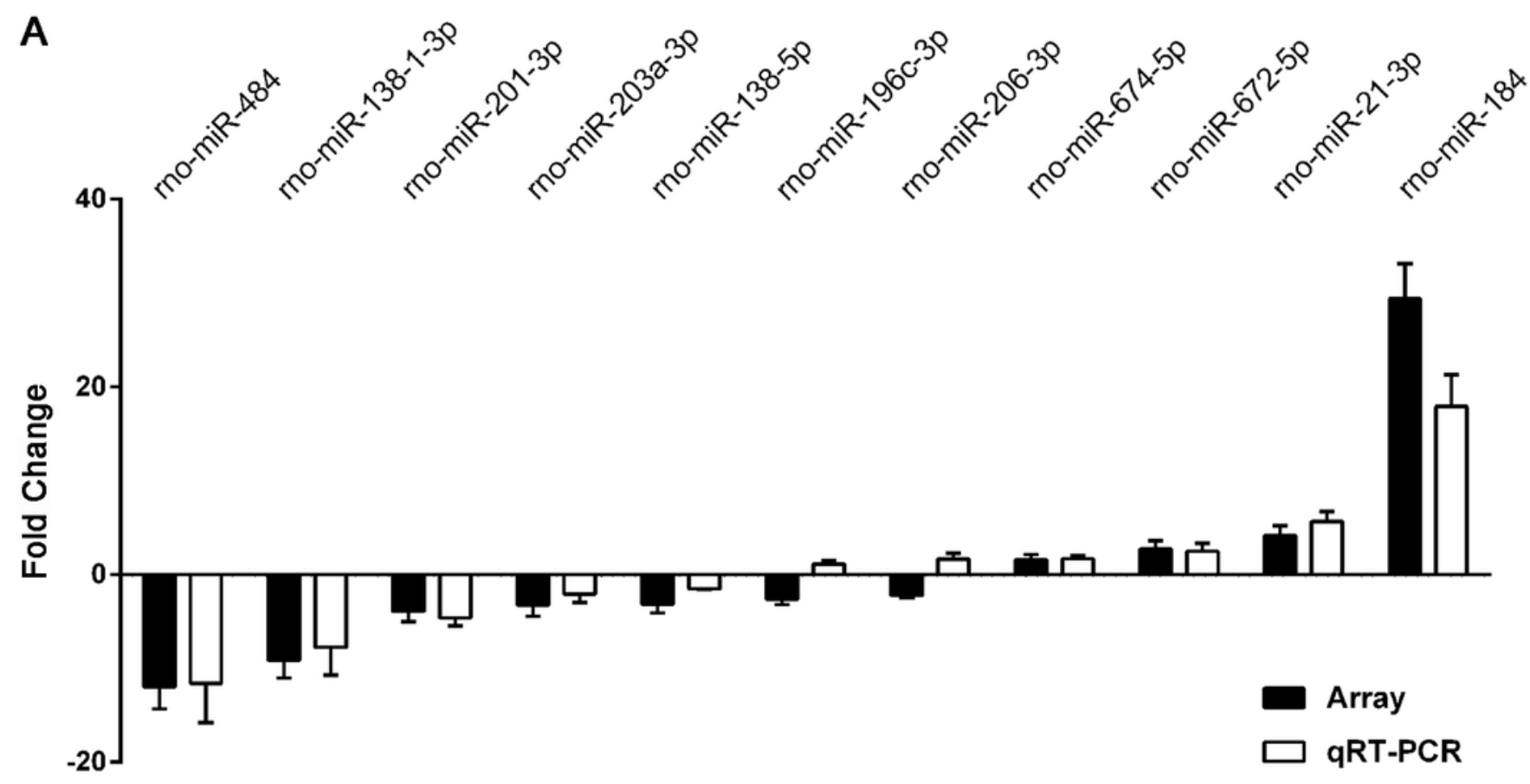

B

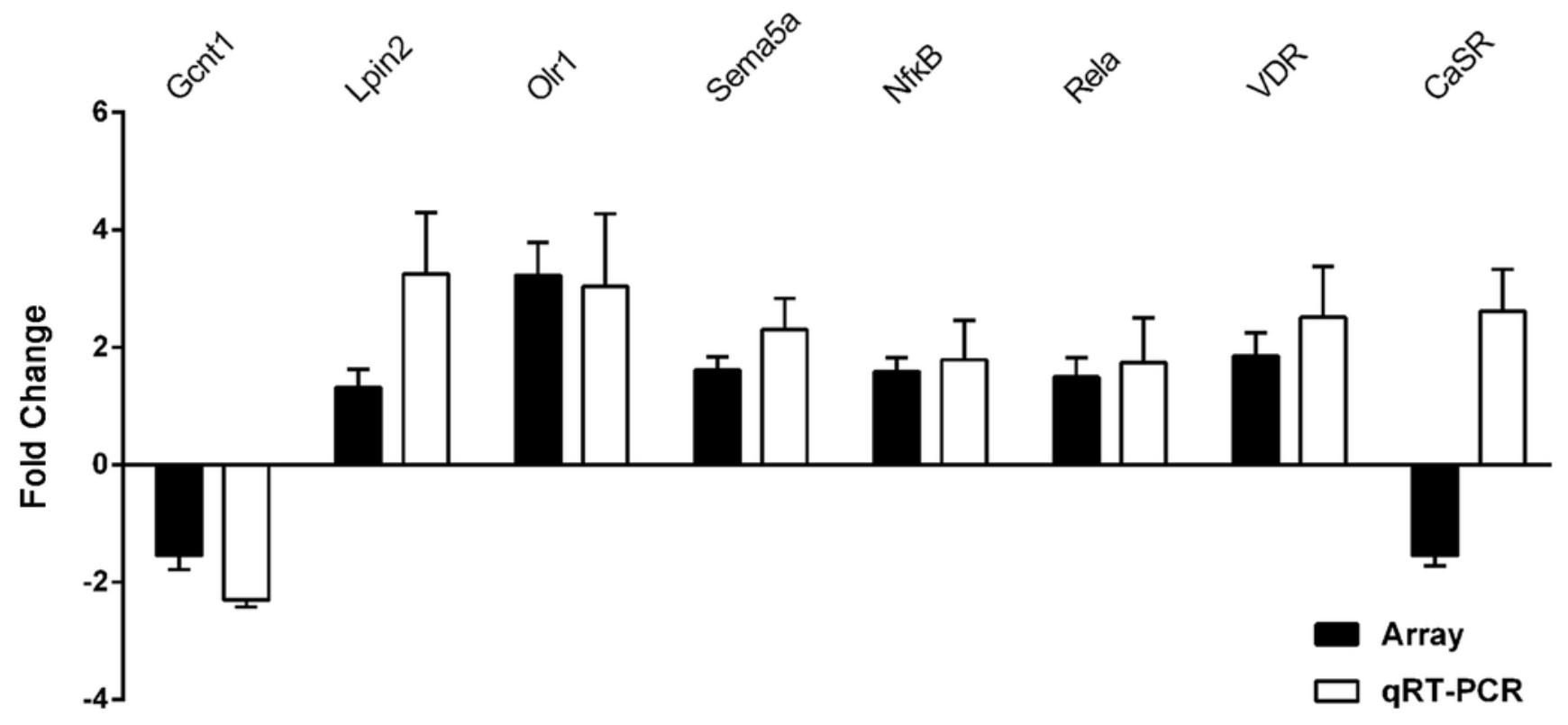

